\title{
COMPARISON OF THE FK5 PROPER-MOTION SYSTEM WITH A KINEMATIC DISTRIBUTION FUNCTION
}

\author{
KAVAN U. RATNATUNGA \\ National Research Council \\ Space Data and Computing Division \\ WAYNE H. WARREN Jr. \\ ST Systems Corporation \\ Astronomical Data Center \\ National Space Science Data Center \\ NASA Goddard Space Flight Center \\ Greenbelt, Maryland 207\%1 \\ U. S. A.
}

\begin{abstract}
A model for the kinematic distribution function of our Galaxy can be used as an independent confirmation that a reference system is free of Earth motions and retains the true kinematics of the stars. Maximum likelihood can simultaneously estimate the parameters required to calibrate distances to the stars, represent the kinematic distribution function, and check on residual Earth rotations in the proper-motion system. The global maximum-likelihood analysis uses all available information: photometry, trigonometric parallax, proper motion, and line-of-sight velocity for a well-defined catalog of stars. Awaiting observations from HIPPARCOS, preliminary testing of the algorithm on available ground-based observations is discussed.
\end{abstract}

\section{Introduction}

Ideally, proper motions should be independent of Earth motions and retain the true kinematics of the stars. The direct way to link the reference system realized by FK5 or HIPPARCOS to an inertial coordinate system is to measure extragalactic sources; however, these sources have significantly fainter apparent magnitudes than most of the reference stars in these fundamental catalogs.

An independent confirmation that the resulting reference system is inertial can be obtained using a Galaxy model for the kinematic distribution. Line-of-sight velocities for the reference stars are independent of the slow motions of the Earth and sample the same stellar kinematics in our Galaxy as the proper motions.

The currently-adopted 1976 IAU precession constant is based on a Galaxy model that is simple to compute. In most analyses the velocity distribution function was assumed isotropic. However, Eichhorn (1974) warns of the error that the observed anisotropy could introduce into the estimated precession constants.

With currently available computing resources, it is possible to estimate simultaneously both the parameters required to represent the kinematic distribution function as well as to look for systematic errors in the reference system and other observables. The global 
maximum-likelihood analysis uses all available information; photometry, trigonometric parallax, proper motion, and line-of-sight velocity for a well-defined catalog of stars.

We are currently developing the required Numerical Algorithms for Maximum Likelihood Estimation (hereafter referenced by the acronym NAMaLiE) for this analysis. Hopefully, observations from HIPPARCOS will generate a well-defined catalog with sufficient accuracy and uniformity appropriate for detailed statistical analysis. The algorithms are being tested on available catalogs of ground-based observations.

\subsection{ADVANTAGES OF THE NAMaLiE APPROACH}

The classical approach for the analysis is to transform observed quantities to a theoretical reference frame, such as to evaluate a distance from an observed trigonometric parallax, or to compute space velocities using proper motions, line-of-sight velocities, and parallax estimates. The only advantage is that the analysis is simple to compute, but the procedure requires the combination of many different types of observations and errors selected with various criteria. This makes rigorous statistical analysis and investigation of systematic errors impossible.

The likelihood function used in NAMaLiE is defined using the directly observed quantities, which allows for proper handling of errors of observation, sample selection, and censorship. We overcome sample incompleteness by using conditional probabilities, a procedure that discards the 'difficult-to-use' information (Casertano, Ratnatunga \& Bahcall 1990).

NAMaLiE also allows the analysis of samples for which trigonometric parallax, proper motion and line-of-sight velocity are not all available for every star. In magnitude-limited reference catalogs, photometry and proper motions are typically available for most stars, while trigonometric parallaxes and line-of-sight velocities are available only for a subset.

Numerical simulations are used to show that the NAMaLiE gives unbiased estimates for parameters and their associated errors. The procedure is a simple compute intensive approach that does not require any clever analytical solutions. Most analytical solutions require simplifying assumptions and approximations, do not solve the real problem, and may give biased estimates of the parameters.

\section{The Galaxy Model}

The distribution function is represented by a sum of stellar populations, the kinematics of each being represented by a trivariate-shifted Gaussian, as used in the IAS-Galaxy model (Ratnatunga, Bahcall \& Casertano 1989). The kinematic distribution for each population is then represented by six free parameters, consisting of three velocity dispersions and three streaming motions.

The model must also include the parameters required to calibrate stellar distances from their observed photometry and available trigonometric parallaxes, as described in Ratnatunga \& Casertano (1991).

For simplicity, consider a selected sample of stars from a single luminosity class and a limited range of temperature (color) over which the color/absolute-magnitude calibration can be assumed as linear. Assume that the cosmic scatter in absolute magnitude is Gaussian and constant over that color range. The absolute-magnitude calibration can then be represented by three free parameters.

Additional parameters are required to define the density distribution and relative normalizations. For stars in the solar neighborhood, the kinematics and distribution of stars can be assumed as uniform, and a single free parameter is sufficient to define the relative normalization for each extra component. 
The residual systematic error in proper motion can be represented as rotations about the three axes. A systematic error in trigonometric parallax may be represented, for example, as errors in the zero point and in the size of the published error estimates.

Hopefully, the catalog has been generated free of systematic errors in photometry and line-of-sight velocity, which are relatively simpler measurements derived from single observations, rather than the difference of two time-separated observations. The analysis uses the coordinates of the stars only to define the line of sight and, as such, is insensitive to typical errors in coordinates.

For example, a spectroscopically selected sample of $K$ and $M$ dwarfs in the solar neighborhood contains stars forming the thin- and old- disk populations (halo stars being only a small $0.2 \%$ contamination) and we would need a total of 24 free parameters. Some of the parameters associated with the mean streaming motion may be held zero by assuming that the density components don't drift relative to the local standard of rest (LSR) radially or vertical to the galactic plane. Then a value for the peculiar motion of the Sun with respect to the LSR could be derived or adopted if poorly constrained by a particular sample.

\section{Characteristics of the Catalog of Observations}

NAMaLiE can make use of incomplete samples of stars and observations as long as the sample selection characteristics are well defined. However, complete samples are desirable to derive reliable information about relative normalizations of stellar components. Although the best available observations should be analyzed, NAMaLiE can make use of data of any accuracy.

The following are required: good coordinates and reliable photoelectric photometry (apparent magnitude and color) for all stars in the sample, all available proper motions transformed to a single uniform reference system, heliocentric line-of-sight velocities, and trigonometric parallaxes for the stars.

To avoid the need for extra free parameters in the Galaxy model associated with any variation of the density and kinematic distribution as a function of location in the Galaxy, a solar neighborhood sample within, say, $100 \mathrm{pc}$ from the Sun is useful. Available trigonometric parallax estimates are also relatively more accurate, and effects of galactic extinction on the photometry are less important. For young, early-type stars, the distribution (example: Gould belt) or kinematics (example: moving groups) may be nonuniform. In an older, late-type stellar population, inhomogeneities in rate and location of star formation have probably time-averaged to a uniform distribution.

However, precise proper motions for a more distant population of stars give a tighter constraint on the residual rotations of the proper-motion system being estimated to link to an inertial reference system. Another advantage of a more distant population is the space-averaging of the distribution sampled; i.e., independent of any peculiarities in local kinematics. The sample selected for analysis must optimize these diverse characteristics with the parameters that need to be estimated and the available accuracy of the observations.

\subsection{INADEQUACY OF AVAILABLE GROUND-BASED SAMPLES}

The analysis requires a sample with good trigonometric parallaxes as well as proper motions in the FK5 system.

The FK5 and Extension, which was the catalog that we hoped to analyze for this paper, consists primarily of intrinsically bright stars with a sampling distance to about $250 \mathrm{pc}$ from the Sun. Line-of-sight velocities and trigonometric parallaxes are available for a 
subset of the sample. However, the accuracy of the currently published parallaxes and the intrinsic width of the giant branch prevented deriving any meaningful estimates for the residual systematic rotations of the proper-motion system.

The spectroscopically selected Vyssotsky $K$ and $M$ dwarf sample has sufficient trigonometric parallaxes and a much smaller intrinsic width of the main sequence to define the absolute calibration. However, the available proper motions have not as yet been transformed to the FK5 system.

\section{Progress To Date}

Most of the required numerical algorithms have been coded and the solutions converge. The procedure has been applied to the Vyssotsky sample of $\mathrm{K}$ and $\mathrm{M}$ dwarfs. The samples need to be represented by two stellar populations with velocity dispersions and asymmetric drift corresponding to the thin and old disk populations. The two populations appear to have statistically different absolute-magnitude calibrations for main-sequence dwarfs with the old disk stars intrinsically fainter and in the Kron $(R-I)$ color range $[0.35,1.3]$. Detailed results will be published in Ratnatunga \& Upgren (1991). The parallax errors seem to be underestimated by $20 \% \pm 7 \%$. The 'mean' zero point of the published parallaxes could be $3.4 \pm 1.4$ mas.

Using this particular catalog of about 750 nearby disk dwarfs with available proper motions, we find no systematic error in the reference system with an rms accuracy of 6 , 7 and 9 mas per year for rotations about the $\mathrm{U}, \mathrm{V}, \mathrm{W}$ axes, respectively. Although this is not a particularly useful constraint, it reflects the total uncertainty, including those from kinematics and the distance calibration. It is derived entirely from information available for this small sample of stars. Observations from HIPPARCOS of a much larger sample of more distant $\mathrm{K}$ and $\mathrm{M}$ giants should compare favorably with the 0.4 mas per year accuracy achieved for the precession constant using lunar laser ranging (Williams et al. 1990).

\section{Conclusion}

A numerical algorithm for maximum-likelihood estimation has been developed to simultaneously estimate the parameters that define the rotation of the reference system and those required to model the disk kinematics and the absolute-magnitude calibration as a function of color. Two stellar components with anisotropic velocity dispersions are required.

Application of this procedure to a sample of $K$ and $M$ dwarfs shows no significant deviations of the FK5 reference system with an rms accuracy of about 7 mas per year. The procedure will be of more practical importance for this problem after observations from HIPPARCOS generate a larger, well-defined catalog of parallaxes and proper motions with sufficient accuracy and uniformity appropriate for detailed statistical analysis.

\section{References}

Casertano, S., Ratnatunga, K. U., \& Bahcall, J. N. 1990, ApJ, 357, 435.

Eichhorn, H. 1974, Astronomy of Star Positions (New York, Frederick Ungar), p. 92.

Ratnatunga, K. U., Bahcall, J. N., \& Casertano, S. 1989, ApJ, 339, 106.

Ratnatunga, K. U., \& Casertano, S. 1991, AJ, in press.

Ratnatunga, K. U., \& Upgren, A. R. 1991, in preparation.

Williams, J. G., Dickey, J. O., Newhall X X, \& Standish E. M. 1990, A\&A, in press. 